\title{
Optimal Hatch Filter with an Adaptive Smoothing Time Based on SBAS
}

\author{
Xiao Zhang ${ }^{1,2}$ \\ ${ }^{1 .}$ Research Institute of Electronic Science and \\ Technology, University of Electronic Science and \\ Technology of China \\ ${ }^{2}$ Key Laboratory of Integrated Electronic System, \\ Ministry of Education \\ Chengdu, China \\ zhangxiao826@gmail.com
}

\author{
Pan Huang ${ }^{1,2}$ \\ ${ }^{1 .}$ Research Institute of Electronic Science and \\ Technology, University of Electronic Science and \\ Technology of China \\ 2. Key Laboratory of Integrated Electronic System, \\ Ministry of Education \\ Chengdu, China
}

\begin{abstract}
The Hatch filter is a code-smoothing technique using integrated carrier phase observations. It is an averaging filter that can smooth pseudorange measurements with continuous carrier phase. It is well known that if the smoothing window is an averaging constant, the filter diverges because of opposite signs of the ionospheric error in the code pseudorange and carrier phase. To solve the problem, this paper suggests a new algorithm for the optimal Hatch filter whose smoothing window width varies adaptively depending on the ionospheric delay variation and the satellite elevation. We use the ionosphere mesh model of the SBAS to calculate the ionosphere delay variation. Based a single day's GPS data, the position accuracy of the pseudorange using optimal Hatch filter outperforms both the pseudorange calculation using classical Hatch filter and the pseudorange calculation without smoothing.
\end{abstract}

Keywords-Global Navigation Satellite System (GNSS), Hatch filter, SBAS, Carrier Phase, Ionospheric delay, Pseudorange smoothing

\section{INTRODUCTION}

The Satellite Based Augmentation System(SBAS), as a GNSS augmentation system, has been implemented to provide differential correction data and integrity information to users. The differential correction data is calculated by the Base Station with the method of carrier phase smoothed pseudorange differential algorithm.

Most of the GPS receivers are able to provide a general measure of the original data, including pseudorange and carrier phase measurements; it also includes the positioning information. The accuracy of carrier phase measurement is generally two orders of magnitude larger than the pseudorange measurements. Therefore, if the value of the entire circumference and the carrier phase value can be calculated, we can get the non-noise pseudorange data. It's very difficult to calculate the entire circumference of the carrier phase, but the Doppler value is easy to obtain. The Doppler measurement data can be an indirect indicative of the carrier phase changes and it can be used as auxiliary information to smooth the pseudo-code range. Using the carrier phase Doppler data to make pseudo-code range more accurate is called carrier phase smoothing pseudorange differential method.

The common method of smoothing pseudorange with carrier phase measurements is called Hatch filter. The application of this recursive algorithm is Hatch filter. Hatch filter smoothing carrier phase only needs to know the current time and the previous observations. It doesn't require the dynamic model or other sensors. Due to the advantages of the Hatch filter, it becomes very popular in single frequency receiver of the region differential system.

\section{OPtIMAL HATCH FILTER ALgORITHM}

The performance of the Hatch filter is determined by the smoothing window width. However, it is well known that if the smoothing window width is an averaging constant, the filter becomes unstable because of opposite signs of the ionospheric error in the code pseudorange and carrier phase.

The United States Federal Aviation Administration (FAA) recommended the Hatch filter to take 100 -second smoothing window for the 1 second observation epoch. Using the 100second window will obtain a relatively accurate pseudorange value, but this is not the best value.

Under the assumption that the ionospheric error is zero, we use Hatch filter to smooth pseudorange. The more satellite epoch we observe, the more accurate pseudorange data will be. However, the effect of the ionospheric delay on pseudorange and the carrier phase is opposite. Thus, overly wide smoothing window will lead to the filter be unbounded. Therefore, choosing an appropriate width of smoothing window will effectively improve the performance of Hatch filter. To differentiate from the classical Hatch filter, this paper considers the ionosphere delay variation and satellite elevation effect on the filter.

We simulate the optimal smoothing window width's relationship to ionosphere delay variation and the satellite elevation. According to Ref.[7], the noise depends on the satellite elevation:

$$
\sigma(\text { elevation })=x_{0}+x_{1} \cdot e^{\left(E L E / x_{2}\right)}
$$


Figure 1 is the result of the simulation about the Relationship with optimal smoothing window to ionospheric delay and satellite elevation.

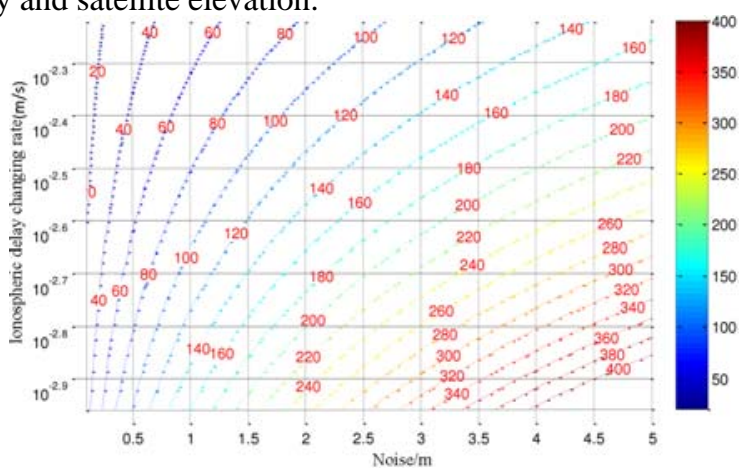

Figure 1 Relationship with optimal smoothing window to ionospheric delay and satellite elevation

Figure 2 is the classic Hatch filter whose smooth window width is a fixed value. Figure 3 shows how we design the optimal hatch filter: we use the optimal smoothing window instead of the fixed window width value. Figure 4 shows how to calculate the optimal smoothing window: we should take the Ionospheric delay variation, the pseudorange noise and the satellite elevation into consideration.

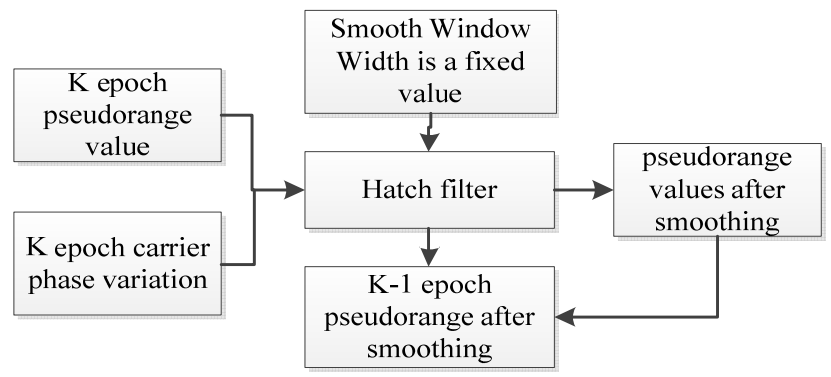

Figure 2 Classic Hatch filter

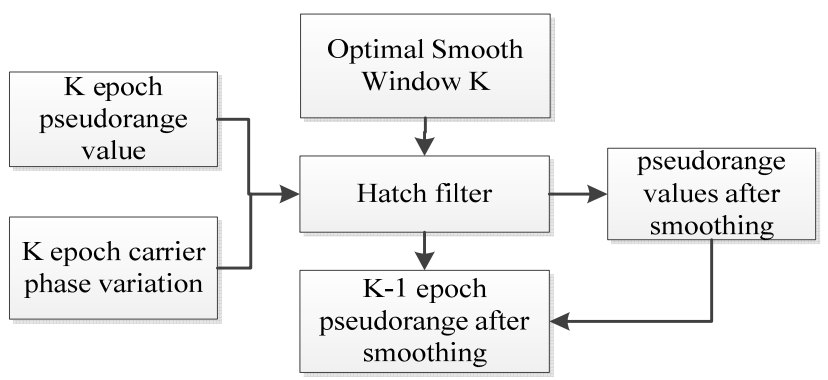

Figure 3 Optimal Hatch filter

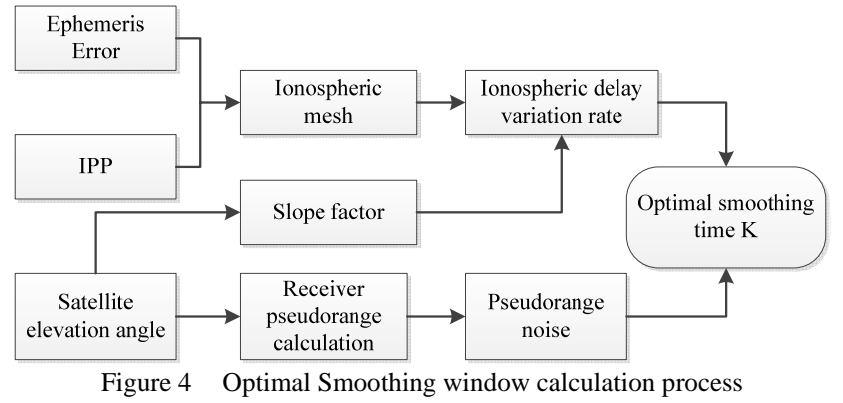

The following process is the calculation of the optimal smoothing window width of the Hatch filter. The observation equation of Hatch filter is:

$$
\left\{\begin{array}{l}
\hat{\rho}(k)=\frac{k-1}{k}[\hat{\rho}(k-1)+\Delta \phi(k)]+\frac{1}{k} \rho(k), k \leq K \\
\hat{\rho}(k)=\frac{K-1}{K}[\hat{\rho}(k-1)+\Delta \phi(k)]+\frac{1}{K} \rho(k), k>K
\end{array}\right.
$$

Where $k$ is the smooth window width of Hatch filter and the maximum of $k$ is $K . \rho(k)$ is raw pseudorange value. $\Delta \phi(k)$ is the carrier phase change in smoothing window. $\hat{\rho}(k)$ is smoothed pseudorange value of the current epoch, $\hat{\rho}(k-1)$ is smoothed pseudorange value of the previous epoch.

If the current epoch is $k$, then the estimated value of the pseudorange can be expressed as:

$$
\begin{aligned}
\rho_{m}(k) & =\rho_{m}(m)+\left(\phi_{k}-\phi_{m}\right) \\
& =R(k)-2 \hat{i}_{\text {dot }}(k-m)+v_{\rho}(m)+v_{\phi}(k)+v_{m}(m) \\
& =\breve{\rho}+v_{\rho}(m)+v_{\phi}(k)+v_{m}(m)
\end{aligned}
$$

Where $\hat{i}_{\text {dot }}$ is the mean value of ionosphere delay variation. $\left(-2 \hat{i}_{d o t}(k-m)\right)$ is the error caused by the ionospheric delay and the observation noise. $R(k)$ is the real distance between the satellite and the receiver. $\breve{\rho}$ is the estimated pseudorange value. $v_{\rho}$ is the pseudorange measurement noise. $v_{\phi}$ is the carrier phase measurement noise.

Hatch filter observes a certain number of epochs and then selects $K$ epochs to smooth the pseudorange. The pseudorange for the last $k$ epochs can be expressed as: 


$$
\begin{aligned}
& \rho_{k}(k)=\breve{\rho}(k)+v_{\rho}(k) \\
& \rho_{k-1}(k)=\breve{\rho}(k)+v_{\rho}(k-1)+v_{\phi}(k)+v_{\phi}(k-1) \\
& \rho_{k-2}(k)=\breve{\rho}(k)+v_{\rho}(k-2)+v_{\phi}(k)+v_{\phi}(k-1) \\
& \ldots \\
& \rho_{k-K+1}(k)=\breve{\rho}(k)+v_{\rho}(k-K+1)+v_{\phi}(k)+v_{\phi}(k-K+1)
\end{aligned}
$$

The mean value of equation (3)is:

$$
\begin{aligned}
\hat{\rho}(k)= & \breve{\rho}(k)+\frac{1}{K}\left\{v_{\rho}(k)+v_{\rho}(k-1)+\cdots+v_{\rho}(k-K+1)\right\} \\
& +\frac{1}{K}\left\{(K-1) v_{\phi}(k)+v_{\phi}(k-1)+\cdots+v_{\phi}(k-K+1)\right\}
\end{aligned}
$$

The noise of the pseudorange is generally high, while the noise of carrier phase measurement is small enough to be ignored. So we can estimate the noise value as:

$$
\begin{aligned}
N(k)= & \frac{1}{K}\left\{v_{\rho}(k)+v_{\rho}(k-1)+\cdots+v_{\rho}(k-K+1)\right\} \\
& +\frac{1}{K}\left\{(K-1) v_{\phi}(k)+v_{\phi}(k-1)+\cdots+v_{\phi}(k-K+1)\right\} \\
& \approx \frac{1}{K}\left\{v_{\rho}(k)+v_{\rho}(k-1)+\cdots+v_{\rho}(k-K+1)\right\}
\end{aligned}
$$

Calculation the covariance of the noise:

$$
E\left[N(k)^{T} N(k)\right] \approx \frac{1}{K} \sigma_{\rho(k)}^{2}
$$

The influence of ionospheric delay to pseudorange and the carrier phase delay is of equal absolute value. But it's opposite:

$$
\begin{aligned}
\rho(k) & =\frac{K-1}{K}\{\rho(k-1)+\Delta \phi(k)\}+\frac{1}{K} \rho(k) \\
& =\rho_{\text {ture }}-\frac{K-1}{K} 2 \bar{i}_{\text {dot }}
\end{aligned}
$$

Deformation equation:

$$
\begin{aligned}
\rho(k)= & \frac{K-1}{K}\left\{\rho_{k-2}(k)-\left(\frac{K-1}{K}\right)^{2} 2 \bar{i}_{d o t}-\frac{K-1}{K} 2 \bar{i}_{d o t}-2 \bar{i}_{d o t}\right\} \\
& +\frac{1}{K} \rho(k)
\end{aligned}
$$

Suppose that with epoch changing, the ionospheric delay variation is linear. Then the gradient of $\bar{i}_{d o t}$ is a constant. The error caused by ionospheric delay can be expressed as:

$$
R(k)=-\sum_{i=1}^{k}\left(\frac{K-1}{K}\right)^{i} 2 \bar{i}_{d o t}=-(k-1)\left\{1-\left(\frac{K-1}{K}\right)^{k}\right\} 2 \bar{i}_{d o t}
$$

Since we already know that $\lim _{K \rightarrow \infty}\left(1-\frac{1}{K}\right)^{-K}=e$, therefore when $K$ approaches infinity, ionosphere error can be simplified as:

$$
R(k)=-(K-1)\left\{1-\frac{1}{e}\right\} 2 \bar{i}_{\text {dot }}
$$

Then the estimated value of the pseudorange is:

$$
\hat{\rho}(k)=\rho_{\text {ture }}(k)+N(k)+R(k)=\rho_{\text {ture }}(k)+\delta \rho(k)
$$

The distance root mean square error(DRMS) of the pseudorange error is:

$$
D R M S^{2}=E\left[N(k)^{T} N(k)\right]+R(k)^{T} R(k)
$$

Take the derivative of $D R M S^{2}$ with respect to $K$ :

$$
\frac{d\left(D R M S^{2}\right)}{d K}=2(K-1)\left(1-\frac{1}{e}\right)^{2} 4 i_{d o t}^{2}-\frac{1}{K^{2}} \sigma_{\rho}^{2}
$$

Let $d\left(D R M S^{2}\right) / d K=0$, as $K$ is the natural number, and equation (13) has only one positive root. After the root is determined, the closest positive integer to the root is the optimal smoothing window $K$. The calculation could be simplified to solve the root of the following equation:

$$
K^{3}-K^{2}-\sigma_{\rho}^{2} /\left[8\left(1-\frac{1}{e}\right)^{2} \bar{i}_{d o t}^{2}\right]=0
$$

In order to solve the above equation, we utilize Carl-Dan equation method. It should be noted that only the positive solution is useful:

$$
K_{\text {temp }}=\sqrt[3]{\frac{\left(-q+\sqrt{q^{2}+4 p^{3}}\right)}{2}}+\sqrt[3]{\frac{\left(-q-\sqrt{q^{2}+4 p^{3}}\right)}{2}}+\frac{1}{3}
$$

Where $p=-\frac{1}{9}$ and $q=\frac{2}{27}-\sigma_{\rho}^{2} /\left[8\left(1-\frac{1}{e}\right)^{2} \bar{i}_{\text {dot }}^{2}\right]$. The optimal smoothing width should be an integer and it can be expressed as $K_{\text {opt }}=\left[K_{\text {temp }}\right]$. 


\section{SIMULATION AND ANALYSIS}

In order to analyze the optimal Hatch filter carrier phase smoothing algorithm, we use the August 12, 2013 GPS data and do three simulations, including no-smoothed pseudorange the error in point measurement, classic Hatch filter smoothed pseudorange error in point measurement and optimal Hatch filter smoothed pseudorange error in point measurement. The error includes ionospheric delay, clock and ephemeris error, tropospheric error and system noise.

TABLE I. COMPARISON OF THREE DIFFERENTIAL MODE

\begin{tabular}{|c|c|c|c|c|c|c|}
\hline & \multicolumn{2}{|c|}{ unsmooth } & \multicolumn{2}{c|}{$\begin{array}{c}\text { Classic } \\
\text { Hatch filter }\end{array}$} & \multicolumn{2}{c|}{$\begin{array}{c}\text { Optimal } \\
\text { Hatch filter }\end{array}$} \\
\hline & $\begin{array}{c}\text { Max } \\
\text { error }\end{array}$ & RMSE & $\begin{array}{c}\text { Max } \\
\text { error }\end{array}$ & RMSE & $\begin{array}{c}\text { Max } \\
\text { error }\end{array}$ & RMSE \\
\hline $\begin{array}{c}\text { X-error } \\
\text { (m) }\end{array}$ & 7.223 & 1.563 & 1.34 & 0.342 & 1.125 & 0.305 \\
\hline $\begin{array}{c}\text { Y-error } \\
\text { (m) }\end{array}$ & 4.443 & 1.479 & 2.395 & 0.77 & 1.753 & 0.683 \\
\hline $\begin{array}{c}\text { Z-error } \\
\text { (m) }\end{array}$ & 7.321 & 3.374 & 4.038 & 1.547 & 2.903 & 0.853 \\
\hline $\begin{array}{c}\text { Error in } \\
\text { point(m) }\end{array}$ & 10.315 & 3.817 & 3.835 & 1.549 & 3.798 & 1.164 \\
\hline
\end{tabular}

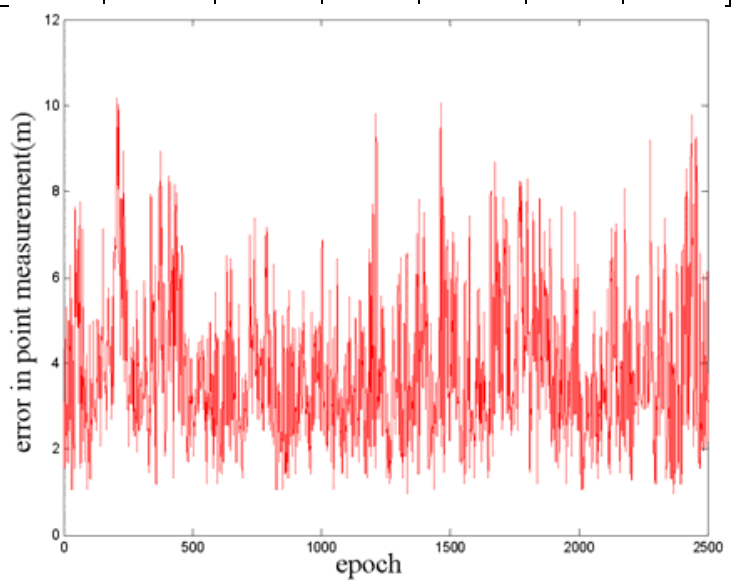

Figure 5 no-smoothed pseudorange error in point measurement

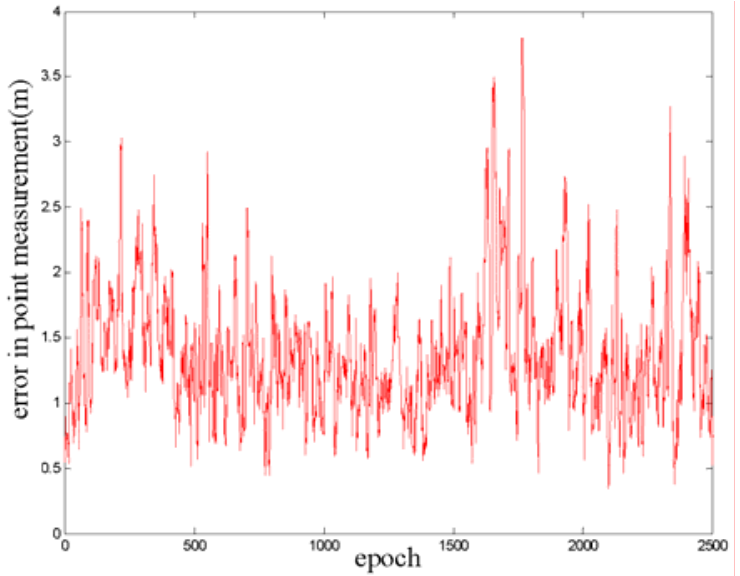

Figure 6 Classic Hatch filter smoothed pseudorange error in point measurement

The error in point measurement can be expressed as:

$$
P E=\sqrt{X_{\text {error }}^{2}+Y_{\text {error }}^{2}+Z_{\text {error }}^{2}}
$$

With respect to the 3 simulation mentioned above, we calculate the accordingly error of the pseudorange with each algorithm, and we also calculate the error in point measurement and the RMSE of them.

From Figure 5 to Figure 7, we can conclude that compared to the no-smoothed pseudorange, the accuracy of classical Hatch filter effectively improved the accuracy of pseudorange and the optimal Hatch filter is better than the classic Hatch filter.

From table 1, we can conclude that the accuracy of no carrier phase smoothed pseudorange differential positioning is very low. When we use classic Hatch filter to smooth pseudorange, the accuracy is improved significantly in $\mathrm{X}$ direction, $\mathrm{Y}$ direction and $\mathrm{Z}$ direction. The accuracy of error in point measurement, when using the optimal Hatch filter, is also improved.

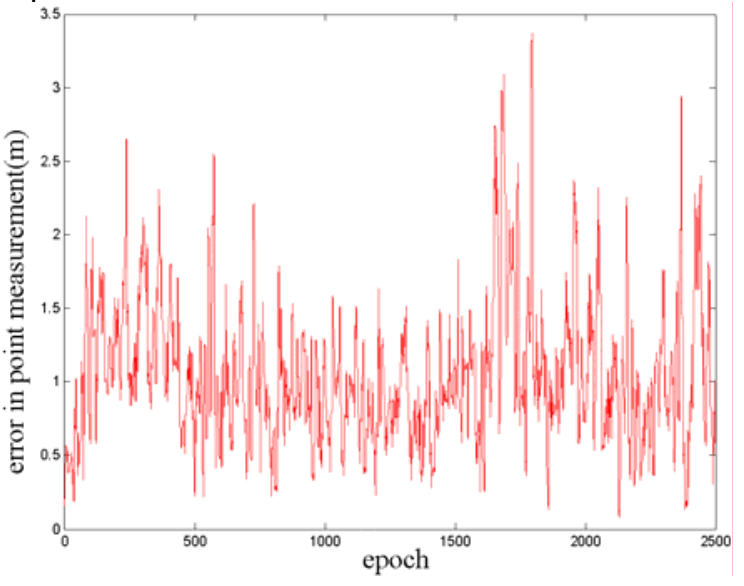

Figure 7 Optimal Hatch filter smoothed pseudorange error in point measurement 


\section{CONCLUSION}

In the pseudorange differential positioning, using Hatch filtering algorithm can improve the positioning accuracy compared to unsmoothed pseudorange. As the optimal Hatch filter has considered the effect of ionosphere delay variation and satellite elevation on smooth window width, its performance is better than the classic Hatch filter. It is one of the contributions of this paper. The precision of the optimal algorithm improvement is about $21 \%$ more than the classical Hatch filter.

\section{REFERENCES}

[1] Hatch R (1983) The synergism of GPS code and carrier measurements. In: Proceedings 3rd

[2] International geodetic symposium on satellite doppler positioning, pp 1213-1231Byungwoon Park,Changdon Kee.Optimal HatchFilter With a Flexible Smoothing Window Width[C].ION
[3] Hwang, P. Y. et al. (1999). "Enhanced DifferentialGPS CarrierSmoothed Code Processing Using Dual-Frequency Measurements.” Navigation: Journal of the Institute of Navigation, Vol. 46, No.2, Summer, pp. 127-137.

[4] Lei, Dengyun, et al. "Carrier-Aided Smoothing for Real-Time Beidou Positioning."Proceedings of the 2012 International Conference on Information Technology and Software Engineering” Springer Berlin Heidelberg, 2013.

[5] Lee, Je Young, et al. "Adaptive position-domain carrier-smoothed code filter based on innovation sequences." (2013).

[6] Xu, Junyi, et al. "Performance Analysis of COMPASS/GPS Code Differential Positioning." China Satellite Navigation Conference (CSNC) 2013 Proceedings. Springer Berlin Heidelberg, 2013.

[7] Kee, C., Park, B., Kim, J., Cleveland, A., Parsons, M., Wolfe, D. (2007). A Guideline on Establishing DGPS Reference Station Requirements, The Journal of Navigation, 61 pp 99-114. 